\title{
Erratum: FOXO3 signalling links ATM to the p53 apoptotic pathway following DNA damage
}

Young Min Chung, See-Hyoung Park, Wen-Bin Tsai, Shih-Ya Wang, Masa-Aki Ikeda, Jonathan S. Berek,

David J. Chen \& Mickey C.-T. Hu

Nature Communications 3:1000 doi: 10.1038/ncomms2008 (2012); Published 14 Aug 2012; Updated 26 February 2013

The immunoblot images in Fig. If of this Article were inadvertently duplicated from panel g during the production process. The correct version of Fig. 1 appears below.

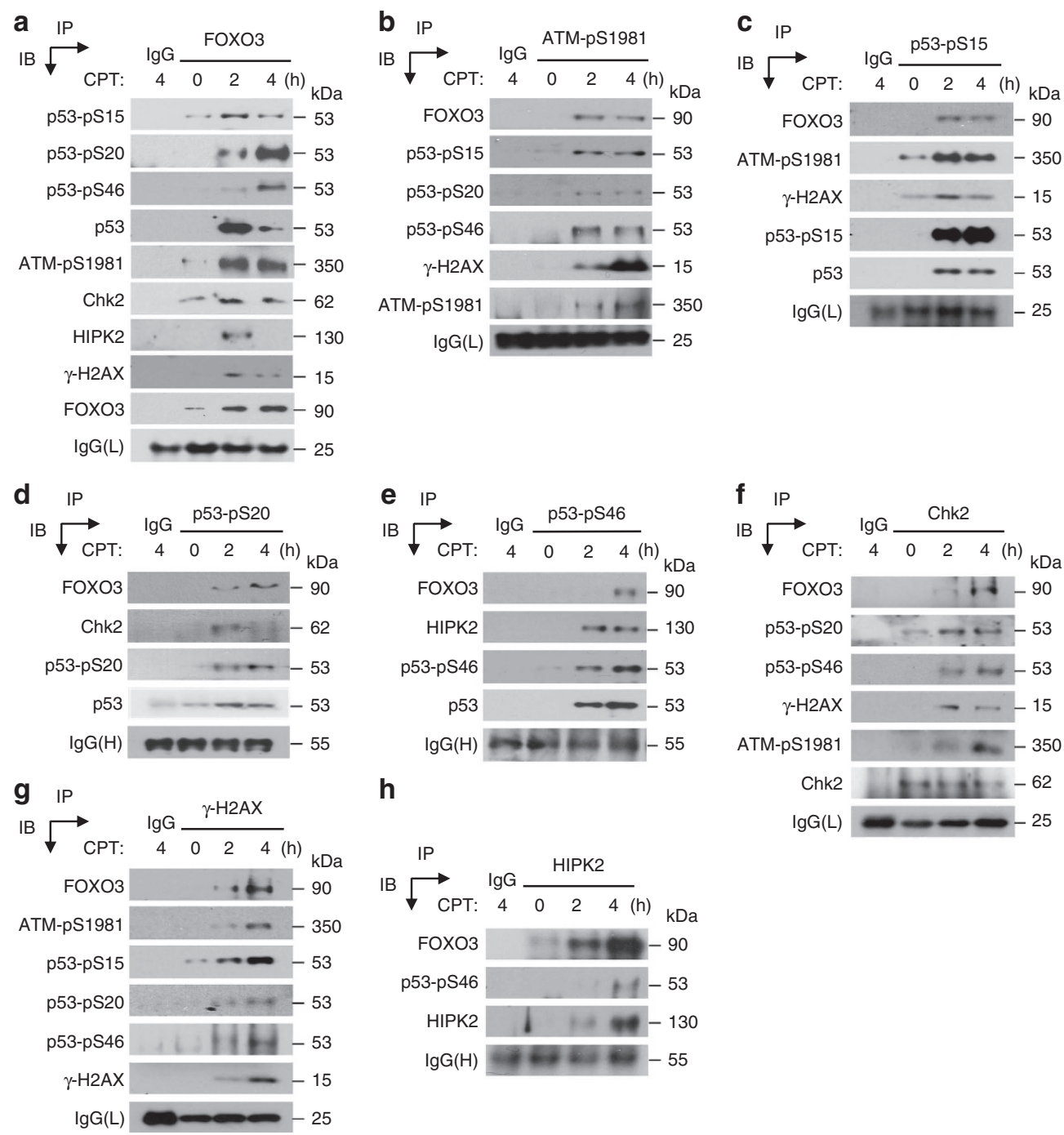

